\title{
Tetrahydropalmatinee alleviates diabetic neuropathic pain by inhibiting the inflammation via p38-MAPK signaling pathway in microglia
}

Lianzhi Cheng ( $\sim 18489961011 @ 163 . c o m)$

Anhui University of Traditional Chinese Medicine https://orcid.org/0000-0003-1892-2583

Junlong Ma

Anhui University of Traditional Chinese Medicine

Aijuan Jiang

Anhui University of Traditional Chinese Medicine

Kai Cheng

Anhui University of Traditional Chinese Medicine

Fanjing Wang

Anhui University of Traditional Chinese Medicine

Qian Chen

Anhui University of Traditional Chinese Medicine

\section{Research Article}

Keywords: Diabetic neuropathic pain, Tetrahydropalmatine, Microglia, p38-MAPK pathway

Posted Date: June 17th, 2021

DOI: https://doi.org/10.21203/rs.3.rs-601230/v1

License: (c) (i) This work is licensed under a Creative Commons Attribution 4.0 International License.

Read Full License 


\section{Abstract}

Object: Exploring the effect of Tetrahydropalmatine (THP) on diabetic neuropathic pain (DNP) and its possible mechanism.

Methods: The type 2 diabetic (T2DM) rat models were prepared by high-fat and high-sugar feeding combined with a single small-dose intraperitoneal injection of streptozotocin (STZ). When the mechanical withdrawal threshold (MWT) and the thermal withdrawal latency (TWL) of T2DM model rats decreased to less than $85 \%$ which were judged as DNP-bearing rats. After treatment with or without THP, the protein expression of hypertonic glycerol reactive kinase (p38), phosphorylated hypertonic glycerolresponsive kinase (p-p38) and OX42 (a specific marker of microglia) were detected by Western Blot and and the mRNA content of p38 and OX42 were detected by qRT-PCR. The expression of pro-inflammatory factors IL-1 $\beta$, IL-6, TNF- $a$, as well as chemotactic factors and their receptors including CXCL1, CXCR2, CCL2 and CCR2 in spinal tissues were detected by ELISA. Serum FINS and GSP content were also detected by ELISA. Double-label immunofluorescence were used to observe the expression of OX42 and p-p38 in the spinal dorsal horn.

Results: Results showed that THP inhibited microglial activation of spinal in DNP rats. And after THP intervention, the MWT and TWL of DNP rats decreased, the expression of p38, p-p38 and OX42 in the spinal cord tissues of rats was significantly reduced while the mRNA of p38 and OX42 also reduced. The expression of IL-1 $\beta$, IL-6, TNF-a, CXCL1, CXCR2, CCL2 and CCR2 in the spinal cord tissues of rats was significantly reduced $(P<0.01)$. At the same time, THP significantly proved FINS, but did not affect FBG and GSP in DNP rats.

Conclusions: THP significantly alleviates pain symptoms in DNP rats, and this effect may be achieved by inhibiting the inflammatory response caused by the activation of microglia mediated by the p38-MAPK signaling pathway.

\section{Introduction}

According to the latest International Diabetes Federation (IDF) investgation in 2019, about $6 \%$ of the world's population is suffering from diabetes, and this number is increasing year by year, the proportion may reach $10 \%$ of the total population by 2045 [1]. What's More, about $20 \%-30 \%$ diabetic patients are sufferring from DNP. The clinical features of DNP are spontaneous pain, paresthesia and hyperalgesia, which seriously affects the physical and mental health of patients [2]. Although the underlying mechanism of DNP is still unclear, there's a growing body of evidence suggestis that the activation of spinal microglia may plays an important role in the occurrence and development of DNP $[3,4]$.

Microglia, the intrinsic macrophages of the central nervous system, are activated in the spinal cord in neuropathic or inflammatory pain(5). It also mediates neuroinflammations and plays an important role in the occurrence and development of DNP [5]. Studies have shown that the spinal cord microglia are activated in STZ-induced diabetic rats $[6,7]$. The activation of microglia is accompanied by the release of 
cytokines and the activation of the p38-MAPK signaling pathway, which is associated with hypersensitivity of inflammatory pain [8]. The phosphorylation activation of p38 leads to the production of pro-inflammatory mediators,such as tumor necrosis factor- $a$ (TNF-a), interleukin 6 (IL-6) and interleukin $1 \beta(\mathrm{IL}-1 \beta)$ etc, leading to hypersensitivity and exacerbating pain symptoms $[9,10]$.

THP is the main bioactive components of the Chinese herbal medicine Corydalis Yanhusuo, which has been widely used for treating pain and cardiovascular disease in traditional Chinese medicine. Clinical and basic science researches have proved that it has good therapeutic effect in alleviating pain [11-13]. Studies have found that THP can specifically block the activation of the p38-MAPK signaling pathway, and thus inhibit the inflammatory response in human monocyte cells [14]. So, can THP alleviate the pain symptoms of DNP rats, and what is the possible mechanism of this effect? Unfortunately, we have not found relevant literature reports. Therefore, we established a DNP rat model to observe the changes in pain threshold, the changes of microglia in the spinal cord, and the p38-MAPK-mediated inflammatory response to explore the effect of THP on DNP and its possible mechanism.

\section{Materials And Methods \\ 2.1 Animals}

80 SPF-grade healthy adult male Sprague-Dawley rats (180-210g) were used, which purchased from Animal Management center of Qinglongshan (Nanjing, China), License No. SCXK20180001, Zhejiang, China. The rats were housed at a temperature of $23-25^{\circ} \mathrm{C}$ and maintained on a 12-hour light-dark cycle (lights on at 7 AM to 7 PM) with 25\%-30\% humidity. And All rats feed and water freely with standard laboratory chow and tap water during experiments. Experiments were performed during the light cycle. All protocols were approved by the Animal Testing Ethics Review Committee of Anhui University of Traditional Chinese Medicine, animal ethics number: AHUCM-rats-2020031.

\subsection{Drugs and reagents}

Tetrahydropalmatine (Molecular formula: $\mathrm{C} 21 \mathrm{H} 25 \mathrm{NO} 4$, Fig. 1) was purchased from Yuanye Biotechnology (S31414, Shanghai, China), which was dissolved in dimethyl sulfoxide (DMSO, PHR13093G, Sigma-Aldrich, St Louis, MO). Streptozotocin (STZ, EZ3414B220, BioFroxx, Germany) powder was dissolved in $0.1 \mathrm{mmol}^{-1} \mathrm{~L}^{-1}$ citric acid solution (C1013, Solarbio, Beijing, China), $\mathrm{pH}=4.4$, protected from light, and used within $30 \mathrm{~min}$.

\subsection{Preparation of DNP rats model and drug treatments}

All the rats were randomly assigned to four groups : Blank control group (Blank) $\mathrm{DNNP}$ model group (Model) $\square$ THP therapy group (THP) and Methylcobalamin positive control group (MeCbl), n=15 per group. The model was established by high-fat and high-sugar diet combined with single dose 
intraperitoneal injection of STZ. In detail, rats of Model, THP and MeCbl groups were fed with high-fat and high-sugar (per $100 \mathrm{~g}$ feed: ordinary feed $74.5 \mathrm{~g}$, lard $10 \mathrm{~g}$, sucrose $10 \mathrm{~g}$, egg yolk powder $5 \mathrm{~g}$, cholesterol $0.5 \mathrm{~g}$ ) while blank control group to be fed regularly. After 4 weeks, except the blank control group rats were injected with citric acid solution intraperitoneally, the other rats were injected with STZ solution $35 \mathrm{mg} / \mathrm{kg}$. After 72 hours, blood was collected from the tail vein to measure fasting blood glucose (FBG) and fasting insulin (FINS). And then, calculate the fasting insulin sensitivity index (ISI), ISI $=$ In $(\text { FINS } \times F B G)^{-1}$. A decreased in ISI as well as $F B G \geq 11.1 \mathrm{mmol} \cdot \mathrm{L}^{-1}$, were identified as a T2DM model rats. 2 weeks later, the MWT and TWL of T2DM rats were detected, and both of them fell below the baseline value of $85 \%$ were judged as DNP rats model. And then, drug intervention was given for 6 weeks, THP group rats were treated with THP by gavage according to their weight $(4 \mathrm{mg} \mathrm{kg}$

$\left.{ }^{1}\right)$, MeCbl group rats were treated with mecobalamin $\left(0.175 \mathrm{mg} \mathrm{kg}^{-1}\right)$, while distilled water $\left(10 \mathrm{~mL} \mathrm{~kg}^{-1}\right)$ for the Blank and Model groups(Fig. 2).

\subsection{Determination of rat weight and FBG}

The weight and FBG of rats in each group were measured before treatment and 2, 4 and 6 weeks after treatment (i.e. $0 \mathrm{~W} 2 \mathrm{~W} 4 \mathrm{~W} 6 \mathrm{~W}$ ). After $12 \mathrm{~h}$ of fasting with normal water supply, the rats were weighed and recorded. The tails of the rats were disinfected with alcohol cotton balls, and the FBG of rat tail venous blood was measured by glucose meter (type 5D-2, Yicheng Bioelectronics Technology, Beijing, China.).

\subsection{Mechanical withdrawal threshold (MWT) test}

We placed the rat on the cribriform metal plate, and separated it with a plexiglass cover. Using Von Frey filaments (North coast, USA) with a test range of $0.008 \mathrm{~g}-300 \mathrm{~g}$ to vertically stimulate the right hind paw of the rats after the rat adapts for 30 minutes, and determine MWT by sequentially increasing and decreasing the intensity of the stimulus. When the filaments are bent, rats aviod them by raising their legs or licking their feet are considered positive reactions. The measurement was performed 5 times in a row with an interval of $15 \mathrm{~s}$ between each measurement. The mean of the minimum grams of positive reaction was regarded as the MWT of the rat.

\subsection{Thermal withdrawal latency (TWL) test}

The rats were put into the intelligent hot plate apparatus (type RB200, Taimeng Software, Chengdu , China) preheated to $55^{\circ} \mathrm{C}$, and the timing was carried out at the same time. When the rat is heated and lick its hind feet, the time ends, and the contact time between the rat's hind feet and the hot plate is recorded. And the measurement was repeated three times, each rat was tested once every 15 minutes. The average of the three results was calculated and recorded as the rat's TWL. It's important to note that the whole test process was kept quiet, and cleaning up the possible dirt in the cage in time after the rat is taken out to avoid affecting the accuracy of the next test results . 


\subsection{Western blot analysis}

Total protein samples extracted from the spinal cord (L4-L6) were prepared by extraction using RIPA lysis bufer (Sigma, St. Louis, MO, USA). Protein concentrations were determined using the BCA Protein Assay Kit (0828A19, Leagene, Beijing, China).

Tese proteins (30 ug of per group) were subjected to $10 \%$ sodium dodecyl sulfate-polyacrylamide gel electrophoresis (SDS-PAGE). Prestained protein marker (00752915, Termo Scientifc, Waltham, MA, USA) was included on each gel. Protein bands from the gel were electronically transferred onto a polyvinylidene difuoride (PVDF) membrane. The membrane were dipped with $5 \%$ non-fat milk in TBST (Sigma, St. Louis, MO, USA) for $2 \mathrm{~h}$ to block nonspecifc interactions. And then the membranes were incubated with the corresponding primary antibodies: $\operatorname{GAPDH}(1: 2000, A 01020$, Abbkine, Wuhan, China), OX42(1:1000, ab1211, Abcam, MA, USA), p38(1:2000, ab170099, Abcam, MA, USA), pp38 (1:800, ab4822, Abcam, MA, USA) overnight at $4{ }^{\circ} \mathrm{C}$. After washing, the membranes were incubated in peroxidase-labeled secondary antibody (mouse/rabbit anti-Rat IgG, 1:2000, A25022/A25020, Abbkine, Wuhan, China) for $2 \mathrm{~h}$. The protein bands were visualized using ECL system (K22030, Abbkine, Wuhan, China) and the intensities were analyzed by the gel imaging device (FCM, ProteinSimple, CA, USA).

\section{8 qRT-PCR analysis}

Total RNA was extracted from the spinal cords using TRIzol reagent (Thermo Fisher Scientific, USA). A NanoDrop ND-300 spectrophotometer (Aosheng, Hanzhou, China) was used to assess the concentrations of extracted RNA. cDNA was synthesized by PrimeScript RT reagent Kit (TaKaRa, Dalian, China). After initial denaturation at $95^{\circ} \mathrm{C}$ for $3 \mathrm{~min}$, amplication was performed for 40 cycles at the following temperature; denaturation at $95^{\circ} \mathrm{C}$ for $5 \mathrm{~s}$; annealing at $56^{\circ} \mathrm{C}$ for $10 \mathrm{~s}$; extension at $72{ }^{\circ} \mathrm{C}$ for $25 \mathrm{~s}$. These were then analyzed by qRT-PCR (CFX-Connect 96, Bio-Rad, Hercules, USA) using SYBR FAST qPCR Master kit (KM4101, KAPA Biosystems, Wilmington, USA) .

The following thermocycling conditions were used: Initial denaturation at $95^{\circ} \mathrm{C}$

And the relative mRNA expression levels were quantifed using the $2-\Delta \Delta \mathrm{Cq}$ method. Primers for $\mathrm{p} 38,0 \mathrm{X} 42$, $\beta$-actin were obtained from Tianyihuiyuan (Wuhan, China). The sequences were following: $p 38$, forward, 5'-AGCAACCTCGCTGTGAATG-3' , reverse, 5' -ACAACGTTCTTCCGGTCAAC-3'; 0X42, forward, 5'CAAGGAGTGTGTTTGCGTGTC-3' , OX42, reverse, 5'-TGAGTATGCCGTTCTTTGTTTC-3'; $\beta$-actin, forward, 5' - CGTTGACATCCGTAAAGAC -3' , $\beta$-actin, reverse, 5' - TAGGAGCCAGGGCAGTA-3' .

\subsection{ELISA analysis}


The blood was put into the non-anticoagulation tube, quiescenced in room temperature for 2 hours, and taking the supernatant after centrifugation at $3000 \mathrm{rpm}$ for 15 minutes. And we taken the L4-L6 spinal cord tissue of the rats, grind it with sterile PBS, centrifuge to take the supernatant. Using the ommercial ELISA Kit (Yiyan Biotechnology, Shanghai, China) to detect the content of FINS and GSP in serum, the contents of IL-1 1 , IL-6, TNF-a, CCL2, CCR2, CXCL1 and CXCR2 in spinal cord tissue. We followed the per manufacturer's directions strictly.

\subsection{Double-labelled immunofluorescence}

Spine cord tissues were fixed in $4 \%$ paraformaldehyde (PFA, Solarbio, Beijing, China) and dehydrated in $30 \%$ sucrose solution overnight for $4{ }^{\circ} \mathrm{C}$. After embedding into OCT compound (4583, SAKURA OCT, USA), tissues were cut into $16 \mu \mathrm{m}$ section. Sections were blocked for $1 \mathrm{~h}$ at room temperature (RT) in blocking solution consisting of

$5 \%$ normal goat serum in PBS containing $0.1 \%$ Triton X-100 (PBST). Primary antibody specific to OX42 (1:100) and p-p38 (1:50) were incubated overnight at $4{ }^{\circ} \mathrm{C}$. The following day, Cy3 or FITC conjugated secondary antibody were incubated for $2 \mathrm{~h}$ at RT. DAPI (C1002, Beyotime, China) was used to stain the nucleus in. The images were acquired using a fluorescence microscope (BX53 biological microscope, Olympus, Japan).

\subsection{Statistical methods}

GraphPad Prism 8.01 (GraphPad software, USA) was used to analyze the relevant data. The results are

expressed in the form of mean \pm standard deviation ${ }^{(\overline{\mathrm{x}} \pm \mathrm{s})}$. All experiments were carried out at least three times. When comparing between groups, if the data satisfies the normal distribution, choosing the oneway analysis of variance (ANOVA). The Kruskal-Wallis rank sum test is used if the data is not satisfied. Multiple comparisons adopting the Fisher's least significant difference (LSD). $P<0.05$ was considered to be statistically significant, $P<0.05$ was considered to be significant statistical significance.

\section{Results}

\subsection{THP ameliorated the weight loss and FINS rise in DNP rats but did not affect FBG and GSP}

Results show that compared with the blank control group, the weight of rats in the remaining groups was significantly higher at $\mathrm{OW}$ and there was no significant difference between those three groups. After 2 weeks of with or without treatment, the weight of rats in those three groups dropped to the lowest level and then gradually increased. Compareing with the model group, the weight of rats in the THP therapy group increased significantly at 4 and 6 weeks of treatment (Fig. 3A). Which indicates that THP can slow 
down the weight loss of rats due to DNP. In addition, comparing with the blank group, the serum levels of FINS in the model group increased significantly, after 6 weeks of treatment, the serum levels of FINS in the therapy group decreased significantly (Fig. 3B), indicating that THP could slow down the rise of FINS in DNP rat. However, THP had no significant effect on FBG and GSP in rats with DNP (Fig. 3C, D)

\subsection{THP ameliorated pain symptoms in DNP rats}

The results show that there were no significant changes in MWT or TWL of the blank group at each time point. But, the MWT and TWL values in the other groups were significantly decreased before treatment (OW) compared with the blank group, and there was no significant difference among those three groups. Meanwhile, the MWT and TWL of the THP therapy group and metacobalamin positive group significantly increased at $2 \mathrm{~W}$ and $6 \mathrm{~W}$, which were significantly higher than those in the model group, indicating that THP had obvious analgesic effect on DNP rats(Fig. 4).

\subsection{THP suppressed inflammation in DNP rats}

The expression levels of IL-1 $\beta$, TNF- $a, I L 6, C C L 2, C C R 2, C X C L 1$ and CXCR2 in the spinal cord of rats were detected by ELISA. Comparing with the blank group, the model group were significantly increased. However, the expression levels of these factors were significantly lower after THP or methylcobalamin treatment than in the model group(fig. 5). And these results suggest that THP suppresses the inflammatory response in DNP rats.

\subsection{THP inhibited the activation of microglia and p38- MAPK in the spinal cord of DNP rats}

The expressions of p38, p-p38 and OX42 were significantly changed in both the model group and the therapy group. Western blotting assay results showed that the expression levels of p38, p-p38 and 0X42 in the spinal cord of rats in the model group were significantly higher than those in the blank group (Fig. 6A-C). And qRT-PCR assay shown the similar results (Fig. 6D, E). These results suggested that THP could inhibit the activation of microglia and p38-MAPK in the spinal cord of DNP rats.

\subsection{THP inhibited the activation of microglia and p38- MAPK in the spinal cord horn of DNP rats}

The expression of OX42 and p-p38 in the spinal cord horn of DNP rats and their co-labeling were observed by double-labelled immunofluorescence. After 6 weeks of treatment, the 0X42 (red) and p-p38 (green) staining of the spinal cord horn was significantly increased in the model group compared with the blank group, and the number of co-labeled in microglia was increased too. Comparing with the model 
group, 0x42 and p-p38 staining as well as the number of co-labeled microglia of the spinal cord horn were decreased in the therapy group after treatment with THP. (fig. 7) These results suggest that THP induces a decrease in p-p38 expression and inhibit microglial activation in spinal cord horn of DNP rats.

\section{Discussion}

DNP is a painful and incurable complication in diabetic patients, characterized by increased sensitivity to mechanical and thermal stimuli [15]. We adopted high-fat and high-sugar feeding for 2 months in this study, and then a single intraperitoneal injection of STZ $(35 \mathrm{mg} / \mathrm{kg})$ was used to destroy the islets to simulate insulin resistance and establish a T2DM rats model [16]. After the T2DM model were established successfully, consistenting with the previous study, the MWT and TWL of the most of rats were significantly reduced within 2 weeks, which considered as successful DNP rats model $[2,17]$.

THP is an active natural alkaloid isolated from Corydalis Yanhusuo which has used for treating pain and cardiovascular disease in traditional Chinese medicine $[18,19]$ In neuropathic pain model mice, THP exerts obvious sedative, hypnotic and analgesic effects [20]. Similarly, THP showed an effective antihyperalgesic effect in a mouse model of neuropathic pain induced by oxaliplatin [21]. In the neuropathic pain model which was induced by segmental spinal nerve ligation, THP can relieve chronic inflammation and neuropathic pain in mice [22]. In this study, the weight of DNP rats increased significantly, and the MWT and TWL responses were improved to varying degrees, after 6 weeks of THP treatment. Based on these data, we speculate that THP may be an effective drug for the treatment of DNP, but the potential mechanism of THP remains to be further studied.

CXCL1 is a chemokine that promote both nociceptor and central sensitization via its main receptor CXCR2, which is a promising target for novel analgesic drugs[23].Studies have shown that CC motif chemokine ligand 2 (CCL2) and its receptor CCR2 play a key role in the occurrence and maintenance of neuropathic pain [24]. Intrathecal injection of CCL2 can produce obvious nociceptive and non-inflamed pain behaviors in rats, and this situation can be reversed by intrathecal injection of CCR2 antagonists. Pre-injection of CCR2 antagonist into the intrathecal not only can prevent and relieve nerve pain but also can inhibit the activation of microglia and p38-MAPK [25-27] In addition, studies have shown that in neuropathic pain caused by CCl surgery, the expression of CXC motif chemokine ligand 1 (CXCL1) and its receptor CXCR2 in spinal dorsal horn neurons and DRG are increased, taking CXCR2 antagonists can reverse mechanical allodynia[28]. Further studies have found that CXCR2 and the activation of microglia may be involved in neuropathic pain, and p-p38 and p38-MAPK may be involved in it [29]. We also found that the levels of CCL2, CCR2, CXCL1 and CXCR2 in the spinal cord tissue of the model group increased in this study, and their expression decreased significantly after THP treatment. These results indicate that THP may alleviate the pain symptoms of DNP rats by regulating the corresponding chemokines and their receptors.

As a kind of resident nervous system phagocytes, microglia play an extremely important role in the occurrence and development of DNP along with its activation and corresponding pro-inflammatory 
reactions [30]. Studies have shown that in diabetic rats, microglia in the spinal cord are activated very early and are positively correlated with pain [31]. The increase expression of OX42 in the model group in this study also proved this, and the decreased of OX42 in the therapy group means that the activation of microglia has been inhibited by THP treatment. The persistent hyperglycemia state of diabetes leads to the activation of microglia, the phosphorylation of p38-MAPK and the release of pro-inflammatory factors (including IL-1 $\beta$, IL-6 and TNF-a) [32].In addition, after peripheral nerve damage, spinal cord microglia are activated, leading to the secretion of inflammatory factors. In turn, inflammation can also activate p38MAPK, thereby promote the activation of spinal cord microglia. In animal models of neuropathic pain, inflammatory factors are considered as a key factor in the occurrence and maintenance of hyperalgesia [33]. In this study, consistent with the reported results, we found that the protein levels of p38 and p-p38 in the spinal cord tissue of the model group increased significantly, while the expression of IL-1 $\beta$, IL- 6 and TNF-a increased. What's more, comparing with the model group, the relevant assays in the therapy group were significantly decreased after THP treatment, we speculate that this is the possible cause of THP alleviating the pain symptoms of DNP rats.

A widely accepted view is that OX42 is a cell surface marker for microglia activation, when peripheral nerves are injured, the morphology of microglia changes and the expression of microglia marker OX42 increases [33]. In the lipopolysaccharide (LPS) -stimulated BV2 microglia cells inflammation model and mouse inflammation model, p38-MAPK inhibitor have a significant inhibitory effect on both microglia activation and neuroinflammation [34]. And further research found that p38 activation in spinal microglia plays a key role in mechanical allodynia in rats [35]. In this study, We observed increased expression of the microglial cell marker OX42 in the spinal cord of DNP rats, accompanied by increased expression of p-p38, and an increased number of cells co-labeled with both. Fortunately, after THP treatment, their expression was significantly inhibited, which indicated that THP can reduce DNP by regulating the p38MAPK signaling pathway in spinal microglia.

In summary, our research shows that DNP rats exhibit activation of microglia and activation of the p38MAPK signaling in spinal cord. And we found that THP can improve the pain symptoms of DNP rats, and this effect may be achieved by reducing the inflammatory response and corresponding chemokines and their receptors. In addition, our research shows that THP can down-regulate the expression of OX42 and p-p38 in spinal microglia, thereby reduce the symptoms of DNP. In conclusion, our study revealed for the first time that THP can exert a therapeutic effect on DNP rats by inhibiting the inflammatory response caused by microglia activation through the p38 pathway. It is beneficial to further develop the analgesic effect of THP for clinical application in DNP therapy

\section{References}

1. 2019, IDF Congress. 2019. Shaping the future of diabetes. Diabetes Res Clin Pract (2019), 107954. DOI 10.1016/j.diabres.2019.107954.

2. Zhu, Y. B., G. L. Jia, J. W. Wang, X. Y. Ye, J. H. Lu, J. L. Chen, M. B. Zhang, C. S. Xie, Y. J. Shen, Y. X. Tao, J. Li, and H. Cao. 2020. Activation of CaMKII and GluR1 by the PSD-95-GluN2B Coupling- 
Dependent Phosphorylation of GluN2B in the Spinal Cord in a Rat Model of Type-2 Diabetic Neuropathic Pain. J Neuropathol Exp Neurol (2020), 800-808. DOI 10.1093/jnen/nlaa035.

3. Zhang, T. T., R. Xue, S. Y. Fan, Q. Y. Fan, L. An, J. Li, L. Zhu, Y. H. Ran, L. M. Zhang, B. H. Zhong, Y. F. Li, C. Y. Ye, and Y. Z. Zhang. 2018. Ammoxetine attenuates diabetic neuropathic pain through inhibiting microglial activation and neuroinflammation in the spinal cord. J Neuroinflammation (2018), 176. DOI 10.1186/s12974-018-1216-3.

4. Zychowska, M., E. Rojewska, A. Piotrowska, G. Kreiner, and J. Mika. 2016. Microglial Inhibition Influences XCL1/XCR1 Expression and Causes Analgesic Effects in a Mouse Model of Diabetic Neuropathy. Anesthesiology (2016), 573-589. DOI 10.1097/ALN.0000000000001219.

5. Hughes, A. N., and B. Appel. 2020. Microglia phagocytose myelin sheaths to modify developmental myelination. Nat. Neurosci. (2020), 1055-1066. DOI 10.1038/s41593-020-0654-2.

6. Shayea, A. M. F., A. M. A. Mousa, W. M. Renno, M. S. Nadar, B. Qabazard, and M. H. M. Yousif. 2020. Chronic Treatment With Hydrogen Sulfide Donor GYY4137 Mitigates Microglial and Astrocyte Activation in the Spinal Cord of Streptozotocin-Induced Diabetic Rats. J Neuropathol Exp Neurol (2020), 1320-1343. DOl 10.1093/jnen/nlaa127.

7. Yang, C., J. Gao, B. Wu, N. Yan, H. Li, Y. Ren, Y. Kan, J. Liang, Y. Jiao, and Y. Yu. 2017. Minocycline attenuates the development of diabetic neuropathy by inhibiting spinal cord Notch signaling in rat. Biomed. Pharmacother. (2017), 380-385. DOI 10.1016/j.biopha.2017.07.078.

8. Ito, N., H. Obata, and S. Saito. 2009. Spinal microglial expression and mechanical hypersensitivity in a postoperative pain model: comparison with a neuropathic pain model. Anesthesiology (2009), 640-648. DOI 10.1097/ALN.0b013e3181b05f42.

9. Huang, Q., X. F. Mao, H. Y. Wu, T. F. Li, M. L. Sun, H. Liu, and Y. X. Wang. 2016. Bullatine A stimulates spinal microglial dynorphin A expression to produce anti-hypersensitivity in a variety of rat pain models. J Neuroinflammation (2016), 214. DOI 10.1186/s12974-016-0696-2.

10. Shin, J., Y. Yin, H. Park, S. Park, U. L. Triantafillu, Y. Kim, S. R. Kim, S. Y. Lee, D. K. Kim, J. Hong, and D. W. Kim. 2018. p38 siRNA-encapsulated PLGA nanoparticles alleviate neuropathic pain behavior in rats by inhibiting microglia activation. Nanomedicine (Lond) (2018), 1607-1621. DOI 10.2217/nnm2018-0054.

11. Yu, J., J. Che, L. Liu, F. Yang, X. Zhu, and B. Cao. 2016. Tetrahydropalmatine attenuates irradiation induced lung injuries in rats. Life Sci. (2016), 74-81. DOI 10.1016/j.Ifs.2016.03.056.

12. Deng, Z., X. Weng, Y. Zhao, and J. Gao, and D. Yu. 2020. Amelioration of muscular spasm-induced pain of Guangtongxiao recipe in a non-everted gut sac in vitro model. J. Ethnopharmacol. (2020), 113040. DOl 10.1016/j.jep.2020.113040.

13. Xiao, S., Y. Zhang, P. Song, J. Xie, and G. Pang. 2019. The investigation of allosteric regulation mechanism of analgesic effect using SD rat taste bud tissue biosensor. Biosens. Bioelectron. (2019), 815-823. DOI 10.1016/j.bios.2018.11.046.

14. Oh, Y. C., J. G. Choi, Y. S. Lee, O. O. Brice, S. C. Lee, H. S. Kwak, Y. H. Byun, O. H. Kang, J. R. Rho, D. W. Shin, and D. Y. Kwon. 2010. Tetrahydropalmatine inhibits pro-inflammatory mediators in 
lipopolysaccharide-stimulated THP-1 cells. J. Med. Food (2010), 1125-1132. DOI 10.1089/jmf.2009.1388.

15. Lee, J. Y., H. Y. Choi, C. S. Park, M. K. Pyo, T. Y. Yune, G. W. Kim, and S. H. Chung. 2019. GS-KG9 ameliorates diabetic neuropathic pain induced by streptozotocin in rats. J. Ginseng Res. (2019), 5867. DOI 10.1016/j.jgr.2017.08.004.

16. Sihota, P., R. N. Yadav, S. Poleboina, V. Mehandia, S. K. Bhadada, K. Tikoo, and N. Kumar. 2020. Development of HFD-Fed/Low-Dose STZ-Treated Female Sprague-Dawley Rat Model to Investigate Diabetic Bone Fragility at Different Organization Levels. JBMR Plus (2020), e10379. DOI 10.1002/jbm4.10379.

17. Tang, H. Y., F. J. Wang, J. L. Ma, H. Wang, G. M. Shen, and A. J. Jiang. 2020. Acupuncture attenuates the development of diabetic peripheral neuralgia by regulating P2X4 expression and inflammation in rat spinal microglia. J. Physiol. Sci. (2020), 45. DOI 10.1186/s12576-020-00769-8.

18. Kang, D. W., J. Y. Moon, J. G. Choi, S. Y. Kang, Y. Ryu, J. B. Park, J. H. Lee, and H. W. Kim. 2016. Antinociceptive Profile of Levo-tetrahydropalmatine in Acute and Chronic Pain Mice Models: Role of spinal sigma-1 receptor. Sci Rep (2016), 37850. DOI 10.1038/srep37850.

19. Zhou, Z. Y., W. R. Zhao, W. T. Shi, Y. Xiao, Z. L. Ma, J. G. Xue, L. Q. Zhang, Q. Ye, X. L. Chen, and J. Y. Tang. 2019. Endothelial-Dependent and Independent Vascular Relaxation Effect of Tetrahydropalmatine on Rat Aorta. Front Pharmacol (2019), 336. DOI 10.3389/fphar.2019.00336.

20. Liu, Y. Y., T. X. Wang, J. C. Zhou, W. M. Qu, and Z. L. Huang. 2019. Dopamine D1 and D2 receptors mediate analgesic and hypnotic effects of I-tetrahydropalmatine in a mouse neuropathic pain model. Psychopharmacology (Berl) (2019), 3169-3182. DOI 10.1007/s00213-019-05275-3.

21. Guo, Z., Y. Man, X. Wang, H. Jin, X. Sun, X. Su, J. Hao, and W. Mi. 2014. Levo-tetrahydropalmatine attenuates oxaliplatin-induced mechanical hyperalgesia in mice. Sci Rep (2014), 3905. DOI 10.1038/srep03905.

22. Zhou, H. H., D. L. Wu, L. Y. Gao, Y. Fang, and W. H. Ge. 2016. L-Tetrahydropalmatine alleviates mechanical hyperalgesia in models of chronic inflammatory and neuropathic pain in mice. Neuroreport (2016), 476-480. DOI 10.1097/WNR.0000000000000560.

23. Silva, R. L., A. H. Lopes, R. M. Guimaraes, and T. M. Cunha. 2017. CXCL1/CXCR2 signaling in pathological pain: Role in peripheral and central sensitization. Neurobiol. Dis. (2017), 109-116. DOI 10.1016/j.nbd.2017.06.001.

24. Illias, A. M., A. C. Gist, H. Zhang, A. K. Kosturakis, and P. M. Dougherty. 2018. Chemokine CCL2 and its receptor CCR2 in the dorsal root ganglion contribute to oxaliplatin-induced mechanical hypersensitivity. Pain (2018), 1308-1316. DOI 10.1097/j.pain.0000000000001212.

25. Chun, S., and Y. B. Kwon. 2019. The CCL2 elevation in primary afferent fibers produces zymosaninduced hyperalgesia through microglia-mediated neuronal activation in the spinal dorsal horn. Brain Res. Bull. (2019), 53-59. DOI 10.1016/j.brainresbull.2019.04.014.

26. Kwiatkowski, K., A. Piotrowska, E. Rojewska, W. Makuch, and J. Mika. 2017. The RS504393 Influences the Level of Nociceptive Factors and Enhances Opioid Analgesic Potency in Neuropathic 
Rats. J Neuroimmune Pharmacol (2017), 402-419. DOI 10.1007/s11481-017-9729-6.

27. Kubickova, L., I. Klusakova, and P. Dubovy. 2020. Bilateral activation of glial cells and cellular distribution of the chemokine CCL2 and its receptor CCR2 in the trigeminal subnucleus caudalis of trigeminal neuropathic pain model. Histochem. Cell Biol. (2020), 239-255. DOI 10.1007/s00418-02001850-4.

28. Manjavachi, M. N., G. F. Passos, G. Trevisan, S. B. Araujo, J. P. Pontes, E. S. Fernandes, R. Costa, and J. B. Calixto. 2019. Spinal blockage of CXCL1 and its receptor CXCR2 inhibits paclitaxel-induced peripheral neuropathy in mice. Neuropharmacology (2019), 136-143. DOI 10.1016/j.neuropharm.2019.04.014.

29. Moraes, T. R., L. S. Elisei, I. H. Malta, and G. Galdino. 2020. Participation of CXCL1 in the glial cells during neuropathic pain. Eur. J. Pharmacol. (2020), 173039. DOI 10.1016/j.ejphar.2020.173039.

30. Chen, J. L., J. H. Lu, C. S. Xie, Y. J. Shen, J. W. Wang, X. Y. Ye, M. B. Zhang, G. L. Jia, Y. X. Tao, J. Li, and H. Cao. 2020. Caveolin-1 in spinal cord modulates type-2 diabetic neuropathic pain through the Rac1/NOX2/NR2B signaling pathway. Am. J. Transl. Res. (2020), 1714-1727.

31. Sun, J. J., L. Tang, X. P. Zhao, J. M. Xu, Y. Xiao, and H. Li. 2019. Infiltration of Blood-Derived Macrophages Contributes to the Development of Diabetic Neuropathy. J Immunol Res (2019), 7597382. DOI 10.1155/2019/7597382.

32. Liu, M., M. Yao, H. Wang, L. Xu, Y. Zheng, B. Huang, H. Ni, S. Xu, X. Zhou, and Q. Lian. 2017. P2Y12 receptor-mediated activation of spinal microglia and p38MAPK pathway contribute to cancerinduced bone pain. J Pain Res (2017), 417-426. DOI 10.2147/JPR.S124326.

33. Dubovy, P., I. Klusakova, I. Hradilova-Svizenska, M. Joukal, and P. Boadas-Vaello. 2018. Activation of Astrocytes and Microglial Cells and CCL2/CCR2 Upregulation in the Dorsolateral and Ventrolateral Nuclei of Periaqueductal Gray and Rostral Ventromedial Medulla Following Different Types of Sciatic Nerve Injury. Front. Cell. Neurosci. (2018), 40. DOI 10.3389/fncel.2018.00040.

34. Gee, M. S., S. W. Kim, N. Kim, S. J. Lee, M. S. Oh, H. K. Jin, J. S. Bae, K. S. Inn, N. J. Kim, and J. K. Lee. 2018. A Novel and Selective p38 Mitogen-Activated Protein Kinase Inhibitor Attenuates LPS-Induced Neuroinflammation in BV2 Microglia and a Mouse Model. Neurochem. Res. (2018), 2362-2371. DOI 10.1007/s11064-018-2661-1.

35. Kishima, K., T. Tachibana, H. Yamanaka, K. Kobayashi, M. Okubo, K. Maruo, and K. Noguchi. 2021. Role of Rho-associated coiled-coil containing protein kinase in the spinal cord injury induced neuropathic pain. Spine J. (2021), 343-351. DOI 10.1016/j.spinee.2020.08.011.

\section{Declarations}

Acknowledgements

Funding: This study was supported by the National Natural Science Foundation of China (No. 8187151923). 
Conflict Of interest: The authors declare that they have no conflict of interest in the publication.

Author Contributions: Aijuan Jiang designed the study. Lianzhi Cheng and Junlong Ma cooperated to complete experimental research and paper writing. Fanjing Wang analyzed the data. Kai Cheng, and Qian Chen modified the manuscript. All authors have seen and approved the manuscript and its contents.

\section{Figures}<smiles>COc1cc2c(cc1OC)C1Cc3ccc(OC)c(OC)c3CN1CC2</smiles>

\section{Figure 1}

Chemical structure of THP. Molecular formula: C21H25NO4.

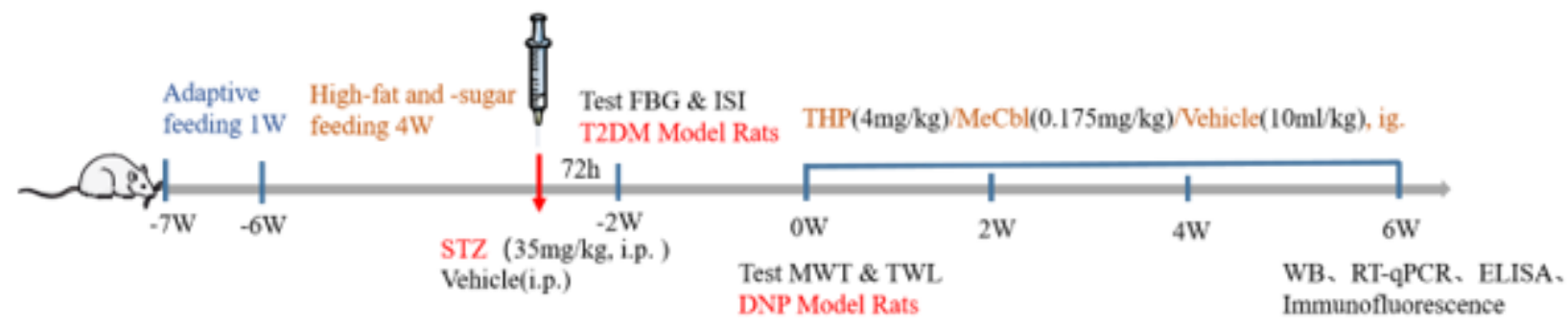

Figure 2

Flowchart of the experiment. 


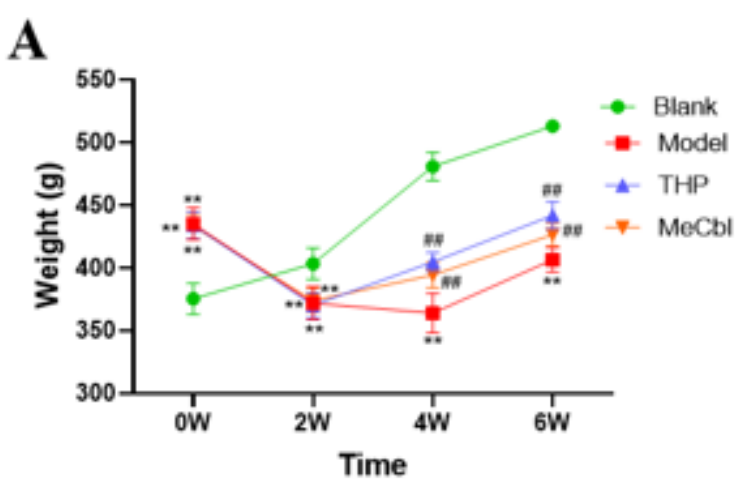

C

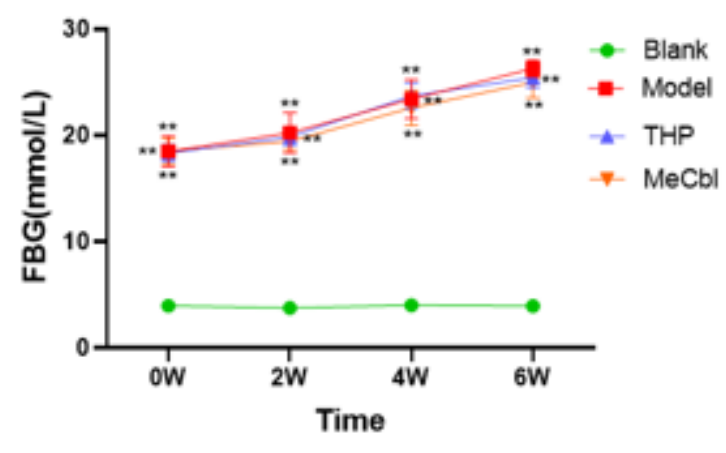

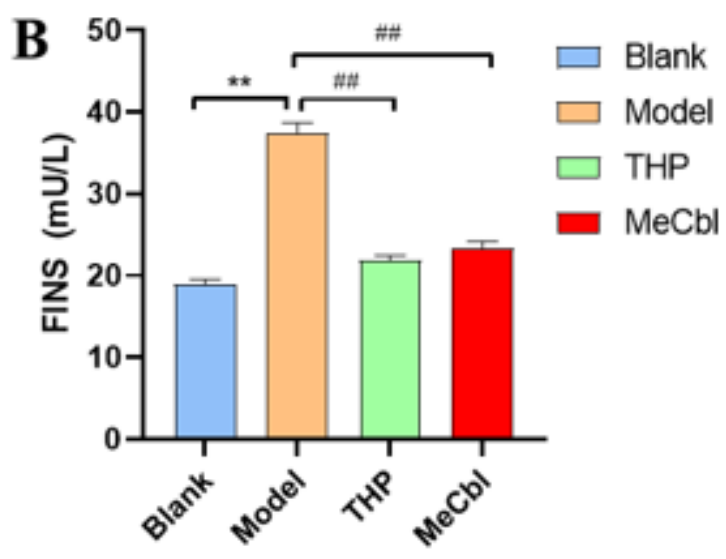

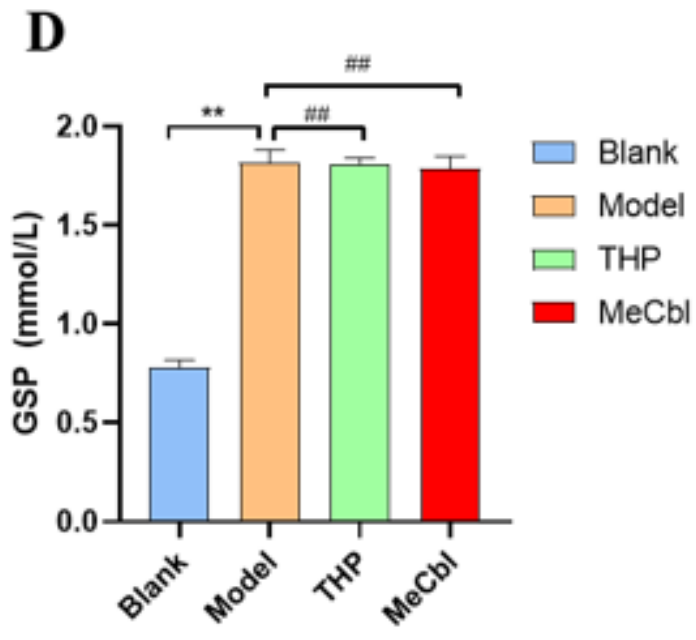

Figure 3

The effect of THP on the weight, FINS, FBG and GSP of DNP rats. A: Weight of rats in each group at each time point. C: Fasting blood glucose (FBG) of rats. D: Serum fasting insulin (FINS) content. E: Glycosylated serum protein (GSP) content. ${ }^{* *} \mathrm{P}<0.01$ compared with the Blank group. \#\# $\mathrm{P}<0.01$ compared with the Model group.

A

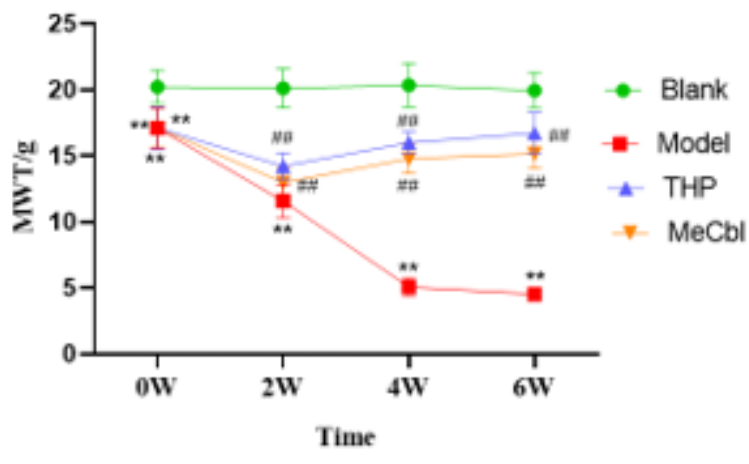

B

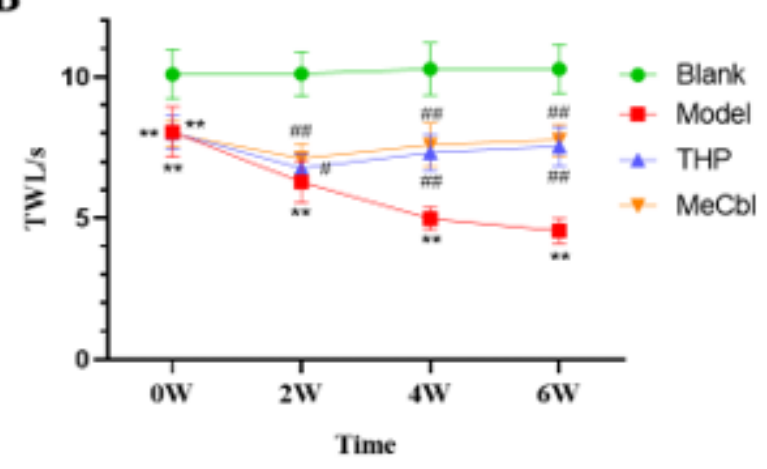

Figure 4 
The effect of THP on pain threshold of DNP rats. A: The mechanical withdrawal threshold (MWT) of rats. $B$ : The thermal withdrawal latency (TWL) of rats. $n=15$ per group. ${ }^{* *} P<0.01$ compared with the Blank group. \# P< 0.05 compared with the Model group. \#\# P< 0.01 compared with the Model group.

A

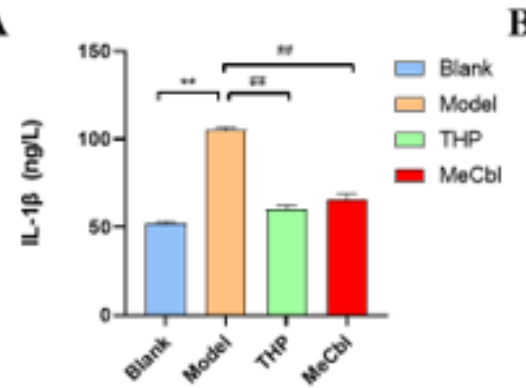

D
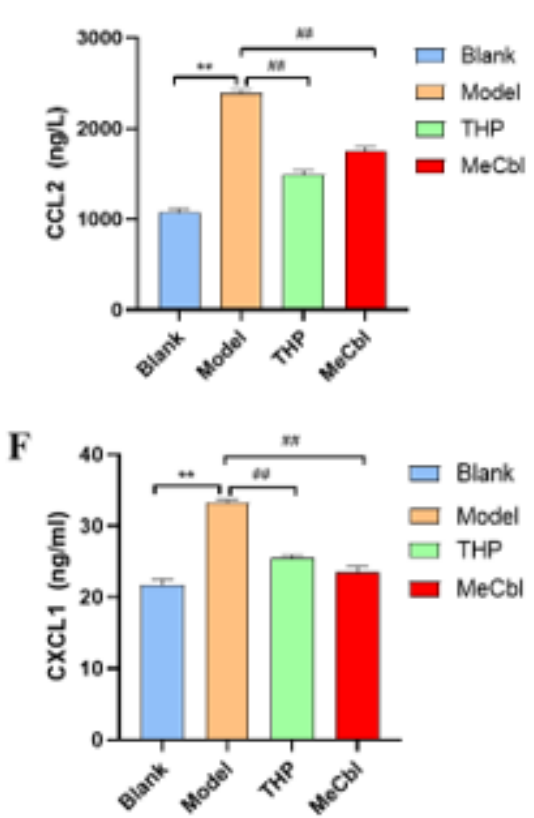

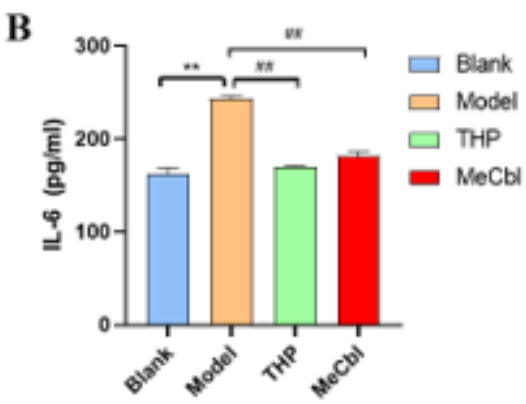

$\mathbf{E}$
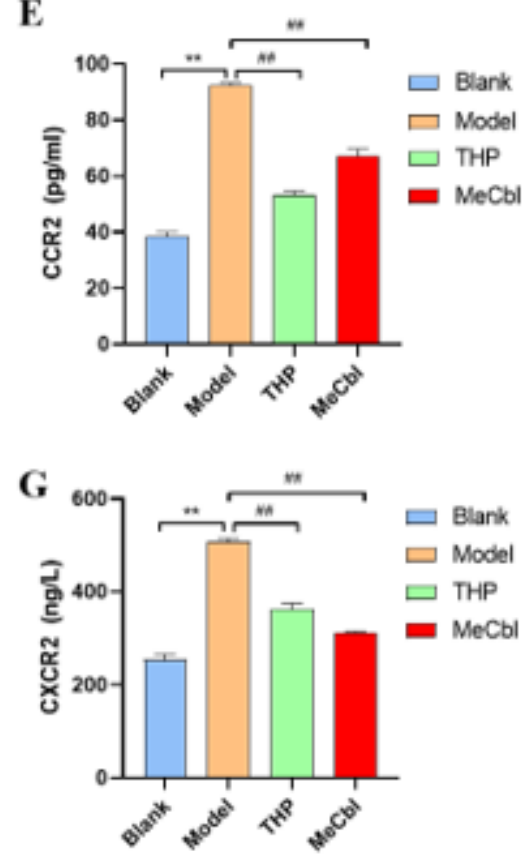

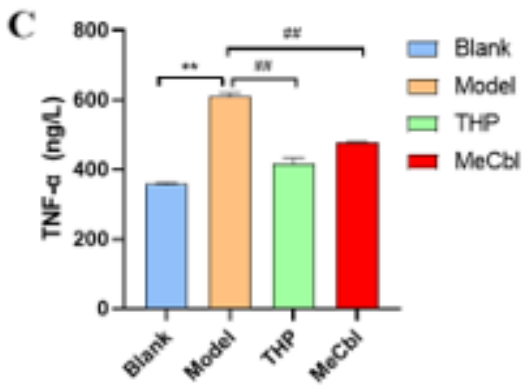

Figure 5

The effect of THP on the inflammation of DNP rats. IL-1 $\beta$ (A) , IL-6 (B) , TNF-a (C) , CCL2 (D) , CCR2 (E), CXCL1 (F) and CXCR2 (G) content in the spinal cord of rats. ** $\mathrm{P}<0.01$ compared with the Blank group. \#\# $\mathrm{P}<0.01$ compared with the Model group. 
A Blank Model THP MeCbl p38

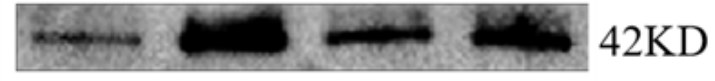

GAPDH

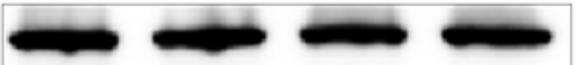

$37 \mathrm{KD}$

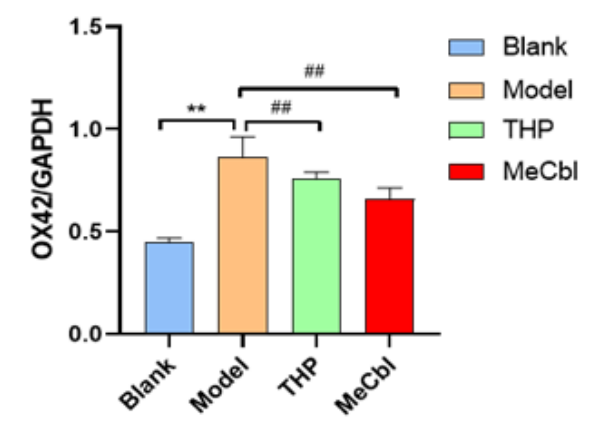

B

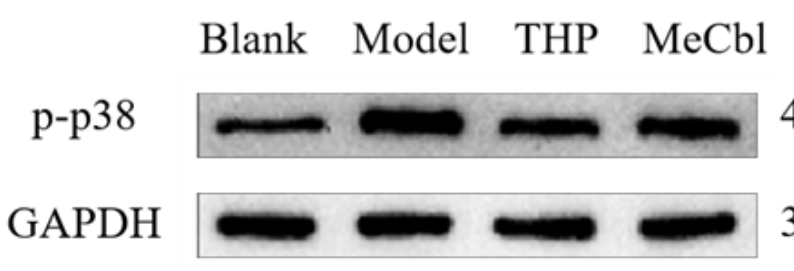
$42 \mathrm{KD}$ $37 \mathrm{KD}$

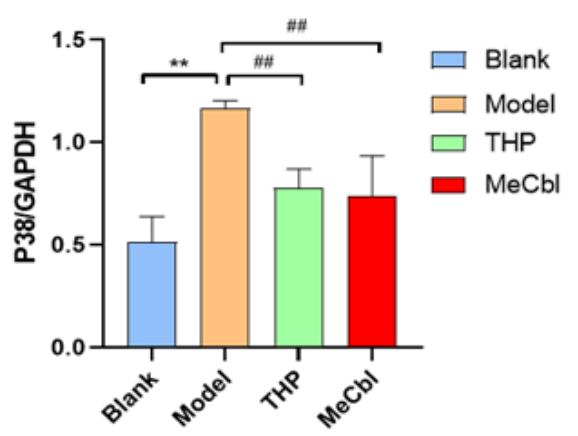

C Blank Model THP MeCbl

OX42

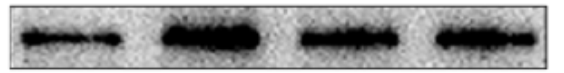

GAPDH

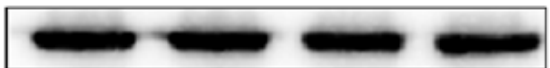

$127 \mathrm{KD}$

$37 \mathrm{KD}$

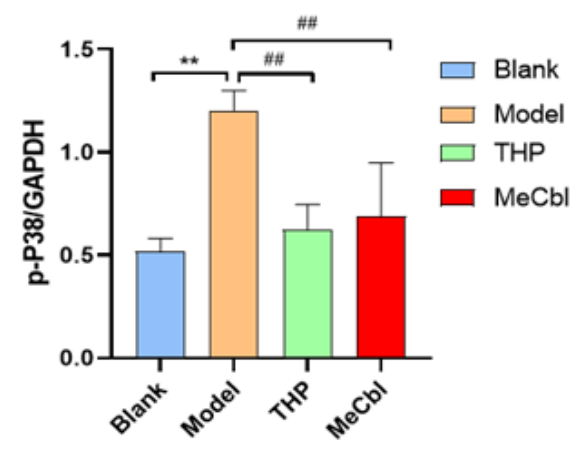

$\mathbf{E}$

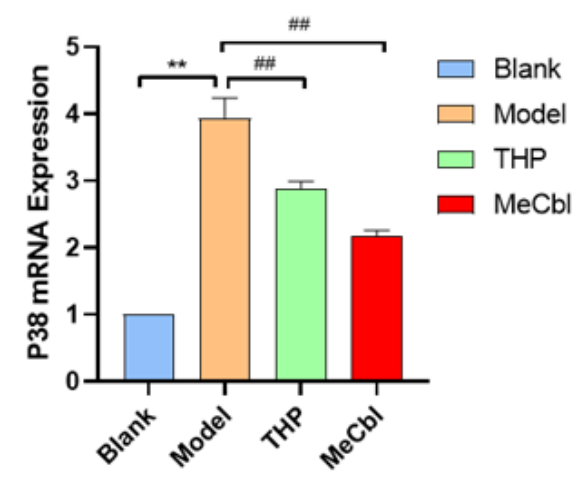

\section{Figure 6}

The effect of THP on the activation of microglia and p38-MAPK pathway in the spinal cord of DNP rats. A: The p38 protein expression in spinal cord of rats. B: The p-p38 protein expression. C: The expression of OX42 protein. D: The mRNA content of p38 in spinal cord of rats in each group. E: The mRNA content of OX42. ${ }^{* *} P<0.01$ compared with the Blank group. \#\# $P<0.01$ compared with the Model group. 


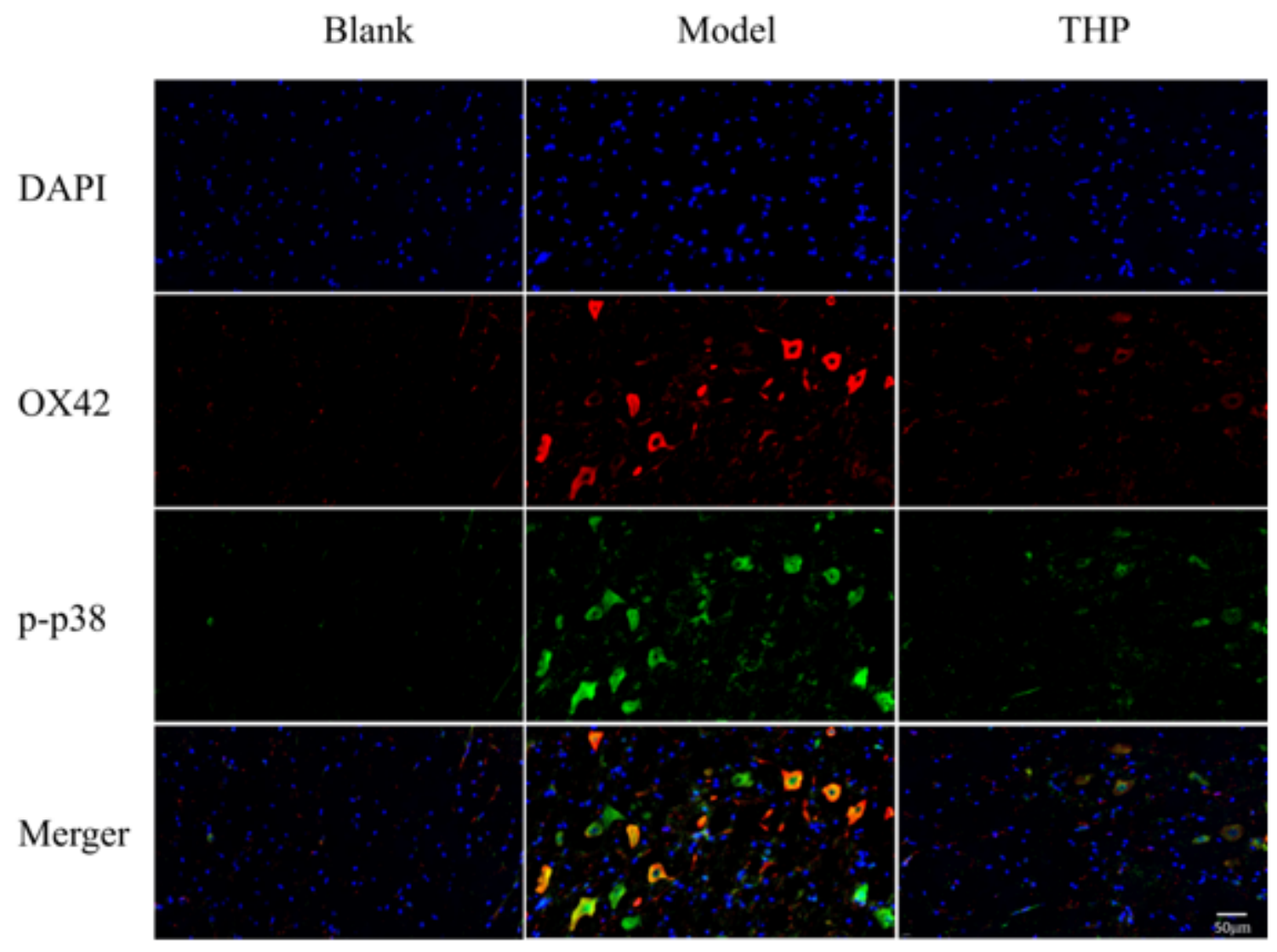

\section{Figure 7}

The effect of THP on the activation and p-p38 expression of L4-L6 spinal dorsal horn microglia in DNP rats. DAPI (blue), p-p38 (green), OX42 (red). \Original magnification $\times 400 \rrbracket$ 